\title{
INFLUÊNCIA DAS DIMENSÕES DA ORGANIZAÇÃO INOVADORA NA GESTÃO DE CUSTOS MEDIADA PELA PASSAGEM EM INCUBADORAS DE EMPRESAS
}

\section{INFLUENCE OF DIMENSIONS OF INNOVATIVE ORGANIZATION ON COST-MANAGEMENT MEDIATED BY PASSING IN BUSINESS INCUBATORS}

0 artigo foi aprovado e apresentado no IX Congreso de Costos del Mercosur, realizado de 28 a 29/11 de 2018, em Montevidéu - Uruguai.

\begin{abstract}
RESUMO
Este estudo teve como objetivo analisar a influência das dimensões da organização inovadora na adoção de práticas de gestão de custos mediada pelo processo de incubação de empresas. Para isso, foram coletados dados a partir de questionário direcionado a uma população de 787 empresas que receberam auxílio financeiro para o desenvolvimento de inovações por meio programas e institutos de apoio ao desenvolvimento de inovações, entre os anos 2009 e 2016. O instrumento englobava as dimensões da organização inovadora (compromisso com a inovação, comunicação, ambiente físico, inovação e tecnologia, pessoas, aprendizagem organizacional, meio ambiente, atuação em equipe e estrutura organizacional), bem como as práticas de gestão de custos (margem de contribuição, custeio variável, custeio por absorção, custo padrão, custos da qualidade, gestão baseada em atividades, kaizen, custeio meta e custeio baseado em atividades), além de outros aspectos, como os relacionados ao processo de incubação. A partir de uma amostra de 97 empresas e do uso de análise descritiva e Modelagem de Equações Estruturais, identificou-se a utilização com maior intensidade de práticas de gestão de custos tradicionais, em detrimento das modernas, com destaque para margem de contribuição. Além disso, não se pode aceitar a hipótese de que a passagem das empresas por incubadoras de empresas poderia mediar a adoção de práticas de gestão de custos em organizações inovadoras, em função da baixa significância do processo de incubação na adoção dessas práticas, do baixo poder explicativo, ou até inverso, que este processo exerce sobre a adoção de tais práticas. Estes resultados podem indicar um gap no suporte, capacitação e consultoria ofertados pelas incubadoras quanto à gestão de custos e suas práticas.
\end{abstract}

Palavras-chave: gestão de custos; inovação; empresas incubadas; organizações inovadoras; práticas de gestão de custos.

\begin{abstract}
This study aimed to analyze the influence of dimensions of the innovative organizations in the adoption of cost management practices mediated by the business incubation process. For this, data were collected using a questionnaire directed at a population of 787 companies that received financial support for the development of innovations through programs and institutes to support the development of innovations, between 2009 and 2016. The instrument encompassed the dimensions innovative organization (commitment to innovation, communication, physical environment, innovation and technology, people, organizational learning, environment, teamwork and organizational structure), as well as cost management practices (contribution margin, variable costing, absorption costing, standard costing, cost of quality, activity-based management, kaizen, target costing and activity-based costing), in addition to other aspects, such as those related to the incubation process. From a sample of 97 companies and the use of descriptive analysis and Structural Equation Model, the most intensive use of traditional cost management practices was identified, to the detriment of modern ones, with emphasis on contribution margin. Furthermore, it cannot be accepted the hypothesis that the passage of companies through incubators could mediate the adoption of cost management practices in innovative organizations, due to the low significance of the incubation process in the adoption of cost management practices, and the low explanatory power and, in some cases, inverse that this process exerts on the adoption of such practices. This result may indicate a gap in the support, training and consultancy offered by incubators regarding cost management and its practices.
\end{abstract}

Keywords: costs management; innovation; incubated companies; innovative organizations; cost management practices.
Fabiana Frigo Souza

Doutoranda em Contabilidade pela Universidade Federal do Paraná (UFPR). Mestre em Contabilidade e Graduada em Ciências Contábeis pela Universidade Federal de Santa Catarina (UFSC). Contato: Av. Prefeito Lothario Meissner, 632, Jardim Botânico Curitiba, PR, CEP: 80.210-170. E-mail: fabiiana_fs@hotmail.com

\section{Valdirene Gasparetto}

Doutora e Mestre em Engenharia de Produção pela Universidade Federal de Santa Catarina (UFSC) Graduada em Ciências Contábeis pela Universidade do Contestado (UNC). Professora associada da Universidade Federal de Santa Catarina (UFSC), atuando no Departamento de Contabilidade, na graduação e nos programas de pósgraduação. Contato: Departamento de Ciências Contábeis, Campus Universitário, Trindade, Florianópolis, SC, CEP: 88.040-900. E-mail: valdirenegasparetto@gmail.com

\section{Simone Bernardes Voese} Doutora e Mestre em Engenharia de Produção pela Universidade Federal de Santa Catarina (UFSC) Graduada em Ciências Contábeis pela Universidade do Vale do Itaja (UNIVALI). Professora associada da Universidade Federal do Parana (UFPR), atuando no Departamento de Contabilidade, na graduação e nos programas de pós-graduação. Contato: Av. Prefeito Lothario Meissner, 632, Jardim Botânico, Curitiba, PR, CEP: 80.210-170. E-mail: simone.voese@gmail.com 


\section{INTRODUÇÃO}

No cenário econômico e competitivo atual, as organizações necessitam aprimorar a execução de suas atividades e o gerenciamento de recursos (Rocha, Souza, Cruz \& Tracz, 2010; Braga, Braga \& Souza, 2010; Bonacin \& Araujo, 2010), e a gestão de custos pode contribuir para o alcance das estratégias e aquisição de vantagens competitivas (Rocha et al., 2010; Uyar, 2010; Raupp, 2011), o que se dá também no ambiente de incubadoras, em que, segundo Raupp (2011), o objetivo é desenvolver os empreendimentos instalados.

Empresas instaladas em incubadoras, sobretudo as de pequeno porte, buscam apoio da incubadora para seu desenvolvimento (Raupp \& Beuren, 2006; Raupp, 2011), incluindo "acesso a instalações físicas subsidiadas, serviços de apoio compartilhado, treinamentos gerenciais, ampliação da rede de contatos, atualização tecnológica e noções sobre os aspectos legais que envolvem os produtos de inovação” (Santa Rita, 2007, p. 17). Assim, incubadoras dão suporte gerencial aos empreendimentos, principalmente nas fases de nascimento ou desenvolvimento (Tiscoski, 2016). Neste sentido, as empresas que passam por processo de incubação têm, nestas fases, suporte para o desenvolvimento de competências gerenciais (Ferreira et al., 2008), por meio do uso de instrumentos de controle gerencial que deem suporte à tomada de decisões (Santos, Dorow \& Beuren, 2016), como as práticas de gestão de custos.

Além disso, outros aspectos possuem relação com a gestão das empresas, como a inovação (Govindarajan \& Trimble, 2006; Arabi \& Kavianifard, 2013; Chenhall \& Moers, 2015), dado que esta não ocorre de forma isolada nas empresas (Bautzer, 2009). Segundo Tidd, Bessant e Pavitt (2008), a inovação precisa de um ambiente propício à sua evolução, a partir do comprometimento com uma cultura de inovação que abranja toda a organização em uma atmosfera criativa, influenciando, portanto, o comportamento de todos e englobando os diversos elementos de uma organização inovadora, como estímulo à criatividade, interesse em inovar, comunicação ampla (Rothwell, 1980; Oliveira, 2006; Tidd et al., 2008), entre outros. Ademais, há indícios de que a inovação influencia positivamente a adoção de novas práticas de gestão de custos como, por exemplo, o Custeio Baseado em Atividades (Baird, Harrison \& Reeve, 2004; Baird, 2007; Charaf \& Bescos, 2013).

A partir do exposto, entende-se que a passagem por um processo de incubação poderá mediar a adoção de práticas de gestão de custos em organizações inovadoras, influenciando, de forma conjunta, a adoção de tais práticas. Neste sentido, considerando o novo cenário onde as empresas estão inseridas, o apoio prestado pelas incubadoras no que diz respeito à gestão dos negócios, bem como o papel da inovação neste processo de gestão, este estudo busca responder a seguinte pergunta de pesquisa: qual a influência das dimensões da organização inovadora na adoção de práticas de gestão de custos mediada pela passagem das empresas pelo processo de incubação? Para responder esta questão-problema, tem-se como objetivo analisar a influência das dimensões da organização inovadora na adoção de práticas de gestão de custos mediada pelo processo de incubação de empresas.

Este estudo justifica-se ao abordar uma lacuna existente na literatura, como mencionado por Santos, Beuren e Conte (2017) e Braga e Raupp (2020), acerca de aspectos gerenciais e incubação de empresas. Além disso, tem-se o espectro das micro e pequenas empresas, maioria entre aquelas que buscam recursos para o desenvolvimento de inovações por meio de projetos, que também merecem atenção da literatura, sobretudo quando se trata do aspecto gerencial (Santos, Beuren \& Conte, 2017). Não obstante, pesquisar empresas com características voltadas à inovação torna-se importante ao analisar que, ainda que representem uma menor parcela das organizações brasileiras, apresentam expressivo faturamento (Iacono, Almeida \& Nagano, 2011). Este estudo está estruturado em cinco tópicos, iniciados por esta introdução. Além disso, tem-se o referencial teórico, os procedimentos metodológicos, a análise e discussão dos resultados e as considerações finais, além das referências.

\section{REFERENCIAL TEÓRICO}

\subsection{Dimensões da organização inovadora}

Tornar-se uma empresa inovadora é um desafio que requer mudanças profundas na estrutura operacional (Costa, 2011). Assim, a organização precisa de uma estrutura de suporte para que a inovação seja possibilitada. Para Volpato e Cimbalista (2002), as pessoas são o elemento central de uma organização inovadora, representando potencial de obtenção de competitividade e fonte de ideias inovadoras. Tidd et al. (2008) afirmam que este contexto é importante para que as ideias criativas possam emergir e ser implementadas.

$\mathrm{Na}$ literatura internacional foram identificados alguns estudos que tratam das características de organizações inovadoras, como aquelas relacionadas aos modelos de Rothwell (1980), Galbraith e Kazanjian (1986) e Tidd et al. (2008). Na literatura nacional, identificou-se o modelo de Oliveira (2006), que engloba os aspectos dos modelos mencionados anteriormente e acrescenta outras dimensões, como ambiente físico, inovação e tecnologia e meio ambiente.

A partir da literatura, percebe-se que aspectos ou características relacionados a organização inovadora convergem entre os autores mencionados, ainda que, em determinados casos, sejam tratados como dimensões distintas. Galbraith e Kazanjian (1986 apud Grant, 2010), por exemplo, inserem a utilização de trabalho em equipe na estrutura, enquanto Tidd et al. (2008) e Oliveira (2006) a consideram como um componente ou uma dimensão relacionada a atuação em equipe.

Há, também, complementariedade entre os estudos mencionados, com a inclusão de novas características para a organização inovadora entre eles. Rothwell (1980) inclui, em seu modelo, a atração de pessoas talentosas e qualificadas, enquanto Galbraith e Kazanjian (1986 apud Grant, 2010) acrescentam o sistema de recompensas, adicionando a percepção 
de que, além de atrair pessoas qualificadas e talentosas, é necessário desenvolver mecanismos para motivar e reter essas pessoas. Tidd et al. (2008), além disso, incluem a necessidade de treinamento e aperfeiçoamento das pessoas e a aprendizagem e disseminação do conhecimento. Oliveira (2006) adiciona, ainda, considerações sobre o ambiente físico onde os colaboradores exercem suas atividades, bem como a preocupação da organização com a preservação do meio ambiente.

Desta forma, para que uma organização seja inovadora, é necessário um contexto propício à inovação, que inclui dimensões e características. A partir desses elementos e dos precedentes da literatura mencionados, entende-se por organização inovadora aquela que possui os aspectos apresentados nas 9 dimensões evidenciadas na Tabela 1, construído como uma síntese dos estudos identificados.

Tabela 1 - Dimensões e características da organização inovadora

\begin{tabular}{|c|c|c|}
\hline Dimensões & Características da organização inovadora & Autores \\
\hline \multirow[b]{2}{*}{$\begin{array}{l}\text { Compromisso com } \\
\text { a inovação }\end{array}$} & Estratégia e gestão voltadas à inovação. & \multirow[b]{2}{*}{$\begin{array}{l}\text { Rothwell (1980); Oliveira (2006); } \\
\text { Tidd et al. (2008) }\end{array}$} \\
\hline & $\begin{array}{l}\text { Foco na qualidade e nos clientes. } \\
\text { Parceria com fornecedores e laboratórios de pesquisa, em busca do } \\
\text { desenvolvimento de inovações. }\end{array}$ & \\
\hline \multirow{3}{*}{ Comunicação } & $\begin{array}{l}\text { Comunicação de forma ampla, onde os colaboradores tenham conhe- } \\
\text { cimento das metas, missão e visão da organização. }\end{array}$ & \multirow{3}{*}{$\begin{array}{l}\text { Rothwell (1980); Oliveira (2006); } \\
\text { Tidd et al. (2008) }\end{array}$} \\
\hline & $\begin{array}{l}\text { Utilização de veículo de comunicação interna e um ambiente em que } \\
\text { as informações possam circular livremente. }\end{array}$ & \\
\hline & Comunicação com a comunidade científica. & \\
\hline Ambiente físico & $\begin{array}{l}\text { Ambiente físico propício ao rendimento do colaborador, sem ruídos e } \\
\text { com espaço adequado para a realização das atividades. }\end{array}$ & Olivera (2006) \\
\hline & Fácil acesso aos recursos materiais necessários às suas atividades. & \\
\hline $\begin{array}{l}\text { Inovação e tecno- } \\
\text { logia }\end{array}$ & $\begin{array}{l}\text { Política de patenteamento. } \\
\text { Investimento em P\&D e estrutura que permita o seu desenvolvimento } \\
\text { (departamento e laboratório de P\&D). }\end{array}$ & Rothwell (1980); Oliveira (2006) \\
\hline & $\begin{array}{l}\text { Estímulo positivo, capacitação, reconhecimento e incentivo formal e } \\
\text { informal. }\end{array}$ & \\
\hline Pessoas & $\begin{array}{c}\text { Abertura para as pessoas se envolverem com a organização, sugerir e } \\
\text { questionar. }\end{array}$ & Oliveira (2006); Tidd et al. (2008) \\
\hline & $\begin{array}{c}\text { Ambiente flexível quanto a horário e local onde as atividades serão } \\
\text { exercidas. }\end{array}$ & \\
\hline $\begin{array}{l}\text { Aprendizagem } \\
\text { organizacional }\end{array}$ & $\begin{array}{l}\text { Compartilhamento de conhecimento dentro da organização, com in- } \\
\text { centivo às pessoas para que compartilhem conhecimentos, experiên- } \\
\text { cias e novas ideias. }\end{array}$ & Oliveira (2006); Tidd et al. (2008) \\
\hline & Atendimento à legislação ambiental. & \\
\hline Meio ambiente & $\begin{array}{l}\text { Preocupação com o meio ambiente como parte do contexto da or- } \\
\text { ganização, produzindo produtos ambientalmente seguros, reciclando } \\
\text { materiais e promovendo a conscientização ambiental }\end{array}$ & Oliveira (2006) \\
\hline Atuação em equipe & O clima organizacional de confiança e espírito de equipe. & $\begin{array}{l}\text { Galbraith e Kazanjian (1986 apud } \\
\text { Grant, 2010); Oliveira (2006); Tidd } \\
\text { et al. (2008) }\end{array}$ \\
\hline $\begin{array}{l}\text { Estrutura organi- } \\
\text { zacional }\end{array}$ & $\begin{array}{l}\text { Controle hierárquico descentralizado, com flexibilidade e aberto a } \\
\text { adoção de novas ideias. } \\
\text { Melhoria contínua dos processos. }\end{array}$ & $\begin{array}{l}\text { Galbraith e Kazanjian (1986 apud } \\
\text { Grant, 2010); Oliveira (2006); Tidd } \\
\text { et al. (2008) }\end{array}$ \\
\hline
\end{tabular}

Fonte: Souza (2017).

A partir do exposto, nesta pesquisa considera-se como organização inovadora aquela que esteja alinhada a aspectos que permitam o desenvolvimento da inovação e, ainda, que possua um ambiente propício para continuidade e expansão, incentivando as pessoas a serem criativas e inovadoras. 


\subsection{Incubadoras de empresas}

Como decorrência da necessidade de uma sociedade baseada em conhecimento (Abib, Hayashi Junior, Gomel \& Fonseca, 2013), a primeira incubadora de empresas surgiu, no Brasil, nos anos 1980 (Iacono et al., 2011). Inicialmente, o foco consistia em setores intensivos em conhecimentos científico-tecnológicos, denominadas incubadores de base tecnológica, no entanto, de forma contemporânea, as incubadoras têm como propósito contribuir para o desenvolvimento setorial e local (Anprotec, 2012).

As incubadoras têm como função principal estimular a criação e o desenvolvimento de micro e pequenas empresas, além da geração de empregos e do desenvolvimento local, oferecendo espaço físico, serviços de treinamento, consultorias e apoio gerencial e técnico, facilitando, ainda, o processo de inovação (Anprotec, 2012; Fernandes, 2014).

Conforme dados da Anprotec (2012), há características comuns entre as incubadoras, como cessão de espaço, oferecimento de serviços básicos, de capacitação e apoio, objetivo de criação de emprego e dinamização da economia, bem como ausência de fins lucrativos. Neste sentido, espera-se que as empresas que passam por um processo de incubação tendam a estar melhor preparadas para inserção no mercado (Raupp, 2011; Lazzarotti, Silveira, Carvalho, Rossetto \& Sychoski, 2015).

Incubadoras de empresas representam um mecanismo que estimula a criação e o desenvolvimento de empresas inovadoras, auxiliando, também, o desenvolvimento econômico regional (Lazzarotti et al., 2015; Broze, Facó, Silva, Souza \& Caten, 2019). Possuem como objetivo desenvolver empresas que, após o período de incubação, sejam financeiramente viáveis e competitivas (Iacono et al., 2011; Abib et al., 2013). Salienta-se, ainda, que as incubadoras podem representar um fator crítico para o sucesso (ou fracasso) das empresas incubadas (Broze et al., 2019).

Incubadoras podem ser divididas entre três grupos, de acordo com o foco das empresas instaladas: (i) incubadoras de economia solidária, formadas geralmente por cooperativas; (ii) incubadoras de base tecnológica, que visam a produção de bens e serviços de alto valor agregado; e (iii) incubadoras com foco em produtos e tecnologias tradicionais (Anprotrec, 2012).

Independente da característica da incubadora à qual pertence a empresa incubada, segundo Raupp (2011), seus gestores precisam considerar as características da gestão de custos, buscando deixá-las adequadas à estrutura operacional e física da empresa, como forma de obter vantagem competitiva a partir de informações e análises.

\subsection{Gestão de custos}

A gestão de custos é utilizada como uma forma de prover informações que deem suporte ao processo de planejamento, controle, desenvolvimento de estratégias e vantagens competitivas (Zhou, Li, Liu \& Song, 2018). Para que a gestão possa atingir estes objetivos, são necessárias práticas que suportem a obtenção de dados e geração de informações (Morais, Coelho \& Holanda, 2014). Várias são as práticas existentes para a operacionalização da gestão de custos, dentre as quais o custeio baseado em atividades (activity-based costing ou $\mathrm{ABC}$ ), a gestão baseada em atividades (activity-based management ou ABM) e o kaizen.

Como forma de identificar as práticas mencionadas recentemente nas pesquisas relacionadas à gestão de custos, fez-se uma busca nas bases de dados Redalyc e Web of Science com os termos "gestão de custos" e "cost management", como delimitação temporal de 2015 a 2018. A partir desses parâmetros foram identificadas as práticas de gestão de custos evidenciadas na Tabela 2 e que têm sido abordadas em pesquisas do período da busca. 
Tabela 2 - Práticas de gestão de custos em pesquisas entre 2015 e 2018

\begin{tabular}{|c|c|c|c|c|c|c|c|c|c|c|}
\hline \multirow[b]{2}{*}{ Autores } & \multicolumn{10}{|c|}{ Práticas } \\
\hline & 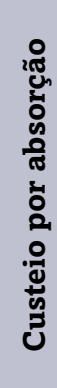 & 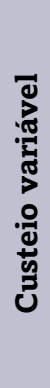 & 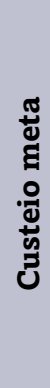 & 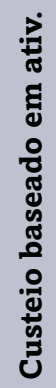 & 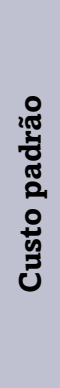 & 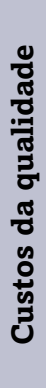 & 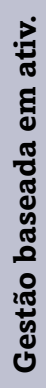 & 芯 & 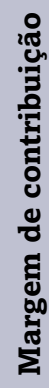 & 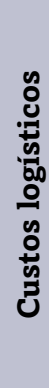 \\
\hline Baharudin e Jusoh (2015) & & & $\mathrm{X}$ & & & & & & & \\
\hline Bokor e Markovits-Somogyi (2015) & $x$ & & & & & & & & & \\
\hline Melo e Leone (2015) & $x$ & $\mathrm{x}$ & & $\mathrm{x}$ & & & & & & \\
\hline Santana, Medeiros e Guimarães (2015) & & & & $\mathrm{x}$ & & & & & & \\
\hline Silva, Gasparetto e Lunkes (2015) & & & & $\mathrm{x}$ & & & & & & \\
\hline Aguilà (2016) & & & & & & & $\mathrm{x}$ & & & \\
\hline Machado, Reckziegel e Souza (2016) & & & & & & & & & & $\mathrm{x}$ \\
\hline Mantri e Jaju (2016) & & & & & & $\mathrm{X}$ & & & & \\
\hline Nowak e Linder (2016) & & & & $\mathrm{x}$ & & & & & & \\
\hline Rezyapova (2016) & & & & $\mathrm{x}$ & & & & & & \\
\hline Silva, Santos Neto, Araújo e Ceolin (2016) & & $\mathrm{X}$ & & & & & & & $\mathrm{x}$ & \\
\hline Vargas, Coser e Souza (2016) & & & & & & & & & & $\mathrm{x}$ \\
\hline Zambon e Bee (2016) & & & & $\mathrm{x}$ & & & & & & \\
\hline Zimon (2016) & & & & & & & & & & $\mathrm{x}$ \\
\hline Ben e Canossa (2017) & & $\mathrm{X}$ & & & & & & & $\mathrm{x}$ & \\
\hline $\begin{array}{l}\text { Engelage, Borgert, Gasparetto, Lunkes } \\
\text { e Schnorrenberger (2017) }\end{array}$ & & & & & & & & & & $\mathrm{x}$ \\
\hline Lima e Azzolini Júnior (2017) & & & & $\mathrm{x}$ & & & & & & \\
\hline Jurčević, Bubalo e Mandžuka (2018) & & & & & & & & & & $\mathrm{x}$ \\
\hline Zhou et al. (2018) & & & & $\mathrm{X}$ & & & & & & \\
\hline
\end{tabular}

Fonte: elaboração própria.

Entre as pesquisas mais recentes, tem-se maior quantidade de estudos relacionados a custeio baseado em atividades. De modo geral, as pesquisas justificam a utilização do ABC com base na premissa de que é uma ferramenta útil para as organizações, gerando informações valiosas para a tomada de decisões (Santana et al., 2015; Nowak \& Linder, 2016; Zambon \& Bee, 2016; Lima \& Azzolini Júnior, 2017), apesar do reconhecimento das dificuldades relacionadas à sua implementação (Santana et al., 2015).

Outra prática com destaque em termos da quantidade de pesquisas identificadas foi a de custos logísticos. Nestes estudos, em geral, o foco tem se concentrado na green logistic, relacionada aos efeitos ou aspectos ambientais da logística (Vargas et al., 2016). No entanto, segundo Wrubel, Diehl, Toigo e Ott (2011), custos logísticos encontram-se no domínio da Gestão Estratégica de Custos e, em decorrência, optou-se por não utilizar a prática no âmbito da gestão de custos, não a considerando, portanto, nesta pesquisa.

No que diz respeito à classificação das práticas de gestão, como as de custos, estas podem ser divididas entre tradicionais e modernas, sendo as primeiras desenvolvidas nos anos iniciais do século XX, enquanto as práticas desenvolvidas mais recentemente são denominadas de modernas (Ifac, 1998; Soutes, 2006; Abdel-Kader \& Luther, 2006). Assim, esta classificação será utilizada nesta pesquisa, tendo como base os estudos de Abdel-Kader e Luther (2006) e Soutes (2006). 


\section{PROCEDIMENTOS METODOLÓGICOS}

\subsection{População e amostra}

A pesquisa foi realizada por meio de levantamento, de abordagem quantitativa, com aplicação de questionário a uma população de 787 empresas que receberam auxílio financeiro para o desenvolvimento de inovações por meio programas e institutos de apoio ao desenvolvimento de inovações, entre os anos 2009 e 2016: Programa Sinapse da Inovação, Instituto SENAI de Inovação, Programa Subvenção Econômica (repassado diretamente às empresas) e Programa TecNova (repassado por meio de fundações de amparo à pesquisa e secretarias e institutos brasileiros). A relação de empresas beneficiadas foi obtida por contato direto ou via site dos órgãos de apoio.

A justificativa para seleção de empresas que tenham recebido apoio financeiro para o desenvolvimento de inovações baseia-se na necessidade de que as organizações, para esta pesquisa, sejam inovadoras em algum grau, ainda que em termos de outputs de inovação, em função de que se pretende buscar nas empresas, a partir da utilização do instrumento de pesquisa, a característica inovadora.

Os dados foram coletados até 10/02/2018 e se obteve o total de 107 respostas, sendo 97 válidas e utilizadas para a análise de dados, representando $12,3 \%$ da população inicial.

\subsection{Instrumento de pesquisa}

O instrumento utilizado foi elaborado a partir do referencial teórico, constituído por quatro blocos que compreendem informações gerais sobre a organização, o respondente, a caracterização da organização inovadora e a utilização de práticas de gestão de custos.

O Bloco 1 (Dimensões da organização inovadora) foi construído a partir da Tabela 1, portanto, da síntese das características e dimensões da organização inovadora identificados até o momento da realização da pesquisa. Cada assertiva foi mensurada por meio de uma escala Likert de 5 pontos, sendo " 1 " discordo totalmente e " 5 " concordo totalmente.

No Bloco 2 (Práticas de gestão de custos), construído a partir de Imlau e Gasparetto (2017), buscou-se identificar a intensidade de adoção das práticas de gestão de custos. Para identificar a intensidade de uso, utilizou-se escala Likert de 5 pontos, sendo "não se aplica" correspondente ao não uso da prática, "1" relacionado à utilização com baixa intensidade e "5" para utilização intensa.

O Bloco 3 (Informações gerais sobre a organização) teve como objetivo caracterizar a organização a partir de informações básicas como nome, quantidade de empregados e setor de atuação, ano de início das atividades e se possui departamento responsável pelas informações contábil-gerenciais. Além disso, neste bloco, as organizações foram questionadas sobre sua passagem ou não por incubadora de empresas.

A pesquisa foi direcionada ao controller das empresas ou à pessoa que exerce função similar. Assim, no Bloco 4 (Informações sobre o respondente), foram questionados os respondentes acerca do seu perfil, como gênero, escolaridade e cargo ocupado, e grau de experiência na atividade.

Antes da aplicação do questionário fez-se uma análise junto a especialistas da área de contabilidade para realização de ajustes necessários à melhor compreensão. Após, realizou-se pré-teste com dois profissionais de contabilidade responsáveis pela geração de informações gerenciais nas empresas onde atuam, com o objetivo de identificar possíveis falhas no instrumento de pesquisa. A partir do pré-teste identificou-se que o questionário abordava os termos de forma clara e precisa, não sendo necessárias outras alterações.

\subsection{Procedimentos para coleta e análise de dados}

Os questionários foram aplicados com a utilização da plataforma Google Forms. Inicialmente os questionários foram encaminhados ao e-mail geral de contato das organizações, obtido nos sites ou fornecido pelas fundações, em seguida, foi feito contato telefônico com as organizações para a obtenção do e-mail direto da pessoa responsável pela elaboração das informações gerenciais. Fez-se, ainda, contato via rede social LinkedIn, partindo da busca pela página das empresas.

A hipótese estabelecida para o estudo foi a de que a passagem das empresas por um processo de incubação pode mediar a adoção de práticas de gestão de custos em organizações inovadoras, influenciando, de forma conjunta, a adoção de tais práticas.

Para a análise, aplicaram-se técnicas de análise multivariada, com a utilização de estatística descritiva e modelagem de equações estruturais (Structural Equation Model - SEM). Os softwares empregados para a análise de dados foram o Excel e o SmartPLS 3.2.7.

Em relação ao uso da modelagem de equações estruturais, justifica-se sua utilização para identificar a intensidade no poder mediador da passagem pelo processo de incubação na relação entre as dimensões da organização inovadora e a adoção das práticas de gestão de custos.

As análises relacionadas ao uso da SEM basearam-se no protocolo de análise evidenciado na Tabela 3. 
Tabela 3 - Protocolo de análise

\begin{tabular}{|c|c|c|c|}
\hline Procedimento & Objetivo & Valor referência & Autor \\
\hline Alfa de Cronbach (AC) & $\begin{array}{l}\text { Avaliar a consistência interna do } \\
\text { constructo }\end{array}$ & Igual ou superior 0,6 & $\begin{array}{c}\text { Hair Jr., Black, Babin, } \\
\text { Anderson e Tatham } \\
\text { (2009) }\end{array}$ \\
\hline $\begin{array}{l}\text { Confiabilidade composta } \\
\text { (CC) }\end{array}$ & $\begin{array}{l}\text { Identificar o grau no qual os itens são } \\
\text { manifestações do construto latente }\end{array}$ & Igual ou superior a 0,7 & Hair Jr. et al. (2009) \\
\hline $\begin{array}{c}\text { Validade convergente } \\
\text { (AVE) }\end{array}$ & $\begin{array}{l}\text { Identificar se os itens que formam o } \\
\text { fator são explicados pelo próprio fator }\end{array}$ & Igual ou superior a 0,5 & Hair Jr. et al. (2009) \\
\hline $\begin{array}{l}\text { Validade discriminante } \\
\text { (VD) }\end{array}$ & $\begin{array}{l}\text { Avaliar se os itens que formam um } \\
\text { fator não estão correlacionados com } \\
\text { outros fatores }\end{array}$ & $\begin{array}{l}\text { Valor da raiz quadrada da AVE } \\
\text { superior ao coeficiente de } \\
\text { correlação entre as variáveis } \\
\text { latentes }\end{array}$ & Hair Jr. et al. (2009) \\
\hline $\mathrm{R}^{2}$ & $\begin{array}{l}\text { Analisar o poder explicativo do } \\
\text { modelo }\end{array}$ & $\begin{array}{l}\text { Quanto maior, maior seu poder } \\
\text { explicativo }\end{array}$ & $\begin{array}{l}\text { Cooper e Schindler } \\
\text { (2004) }\end{array}$ \\
\hline
\end{tabular}

Fonte: elaboração própria.

Antes de iniciar o protocolo de análise, foram analisadas as cargas fatoriais que compõem os construtos do modelo, utilizando como parâmetro os valores acima de 0,5.

\section{ANÁLISE E DISCUSSÃO DOS RESULTADOS}

\subsection{Perfil dos respondentes}

Identificou-se que a maior parte dos respondentes exerce funções de diretor (26\%) e gerente (15\%), com apenas 12 respondentes (12\%) exercendo a função de controller. Além disso, entre os respondentes há cargos como analista contábil e financeiro, sócio administrador, sócio gerente, contador, CEO (chief executive officer), entre outros. Este achado pode indicar que nas organizações analisadas, em geral, não há um profissional da área da controladoria ou que a responsabilidade pela elaboração das informações gerenciais não pertence a este profissional.

A última formação da maior parte dos respondentes (51\%) é em nível de especialização lato sensu e, entre estes, $37 \%$ na área de contabilidade e controladoria. Os demais especialistas possuem formação em outras áreas, como financeira, de gestão, administrativa, de engenharia, entre outros. Nota-se diversidade de áreas e departamentos envolvidos na elaboração de informações gerenciais, com atuação de profissionais de diversas áreas do conhecimento.

\subsection{Perfil das organizações}

Identificou-se que a maior parte das empresas possui menos de 100 funcionários (70\%), o que pode estar relacionado à característica das empresas analisadas, que, em sua maioria, pertencem ao ramo de tecnologia. O porte da empresa foi identificado a partir da metodologia do Sebrae (2013), com base na quantidade de colaboradores, segregando as empresas entre industriais (representando 44\%) e comércio e serviços ( $56 \%$ das empresas).

Entre as empresas industriais, $83 \%$ são microempresas e EPPs, constituídas a partir dos anos 2000, enquanto as de médio e grande porte foram constituídas antes deste período em $90 \%$ dos casos e, portanto, possuem maior tempo de atuação. Quanto às de comércio e serviços, a maioria (78\%) são microempresas e EPPs e foram constituídas, principalmente, a partir de 2005 , representando $64 \%$.

Com relação à passagem das empresas pelo processo de incubação, identificou-se que $64 \%$ não estão ou estiveram instaladas em um ambiente de incubadora de empresas. Entre as que passaram pelo processo de incubação (36\%), a maior parte se constitui de microempresas e EPPs (pouco mais de 80\%), e duas empresas são de grande porte. Este comportamento pode estar relacionado à característica das empresas atendidas pelas incubadoras de empresas que estão, principalmente, em início de atividade e são, portanto, pequenas, buscando suporte para alavancar a sua ideia e seu desenvolvimento, como defendem Raupp e Beuren (2006) e Raupp (2011).

\subsection{Práticas de gestão de custos utilizadas pelas empresas}

No que diz respeito à adoção de práticas de gestão de custos, observa-se a utilização com maior intensidade das práticas tradicionais, todas utilizadas com maior intensidade do que as práticas modernas, conforme evidenciado na Tabela 4. Outras pesquisas encontraram resultados similares, como Joshi (2001), na qual o custeio variável também se encontrava entre as práticas mais utilizadas. Chenhall e Langfield-Smith (1998) identificaram que, em grandes empresas, 
$80 \%$ utilizavam o custeio por absorção. Destaca-se que apenas uma empresa de grande porte, dentre as analisadas, não utiliza essa prática.

Tabela 4 - Classificação das práticas de gestão de custos quanto à média de intensidade de uso e ao percentual de empresas da amostra que utilizam

\begin{tabular}{|c|c|c|c|c|c|c|c|}
\hline \multirow{2}{*}{ Posição } & \multirow{2}{*}{ Prática } & \multicolumn{2}{|c|}{ Classificação ${ }^{1}$} & \multirow{2}{*}{ Média $^{2}$} & \multicolumn{3}{|c|}{ \% de utilização ${ }^{3}$} \\
\hline & & Trad. & Mod. & & $\tilde{\mathbf{N}}$ ou B & $\mathbf{M}$ & $\mathbf{I}$ \\
\hline 1 & Margem de Contribuição & $\mathrm{X}$ & & 3,8 & $7 \%$ & $27 \%$ & $66 \%$ \\
\hline 2 & Custeio Variável & $\mathrm{X}$ & & 3,1 & $23 \%$ & $31 \%$ & $46 \%$ \\
\hline 3 & Custeio por Absorção & $\mathrm{X}$ & & 3,0 & $26 \%$ & $25 \%$ & $49 \%$ \\
\hline 4 & Custo Padrão & $\mathrm{X}$ & & 2,6 & $36 \%$ & $23 \%$ & $41 \%$ \\
\hline 5 & Custos da Qualidade & & $\mathrm{X}$ & 2,6 & $34 \%$ & $29 \%$ & $37 \%$ \\
\hline 6 & Gestão Baseada em Atividades (ABM) & & $\mathrm{X}$ & 2,6 & $29 \%$ & $36 \%$ & $35 \%$ \\
\hline 7 & Kaizen & & $\mathrm{X}$ & 2,5 & $35 \%$ & $28 \%$ & $37 \%$ \\
\hline 8 & Custeio Meta & & $\mathrm{X}$ & 2,4 & $38 \%$ & $27 \%$ & $35 \%$ \\
\hline 9 & Custeio Baseado em Atividades (ABC) & & $\mathrm{X}$ & 1,9 & $47 \%$ & $31 \%$ & $22 \%$ \\
\hline
\end{tabular}

Fonte: elaboração própria.

Legenda: Trad.: Tradicional. Mod.: Moderna; ${ }^{1}$ Classificação de acordo com Soutes (2006) e Abdel-Kader e Luther (2006); ${ }^{2}$ Média de intensidade de utilização calculada com base em escala Likert de 5 pontos, sendo 1 pouca intensidade e 5 utilização intensa; ${ }^{3}$ Percentual de utilização calculado com base na quantidade de empresas que utilizam a prática de acordo com a intensidade e em relação ao total de empresas, sendo: $\tilde{N}$ ou B: percentual de empresas que não utiliza ou utiliza com baixa intensidade; M: percentual de empresas que utiliza com intensidade moderada; I: percentual de empresas que com utilização intensa.

Conforme evidenciado, o custeio baseado em atividades é a prática com menor intensidade de utilização entre as empresas analisadas. Este resultado se assemelha aos obtidos Naranjo-Gil, Maas e Hartmann (2009), os quais identificaram que $31 \%$ das empresas não utilizavam o ABC e $49 \%$ utilizava com baixa intensidade. Como mencionado anteriormente, apesar de esta ser uma prática que gera informações úteis para a tomada de decisões, muitas são as dificuldades relacionadas à sua implementação, o que pode dificultar a adoção desta prática por parte das empresas analisadas.

Destaca-se que estes resultados podem indicar falhas no processo de gestão de custos das empresas analisadas, que é uma realidade vivenviada principalmente em empresas de portes menores, como evidenciado por Raupp (2011) e Braga e Raupp (2020), por exemplo.

\subsection{Análise do modelo de equações estruturais}

A análise do modelo de equações estruturais incluiu a avaliação do modelo de mensuração e do modelo estrutural.

Em relação ao modelo de mensuração, fez-se, primeiramente, a análise das cargas fatoriais, mantendo aquelas que apresentavam valores acima de 0,5. Neste ponto, foram necessárias algumas exclusões, que incluem assertivas das dimensões comunicação, inovação e tecnologia e estrutura organizacional. Quanto ao alfa de Cronbach (AC), fez-se necessária a exclusão da assertiva 2 da dimensão aprendizagem organizacional. A partir dos ajustes, obteve-se confiabilidade acima de 0,6 para todas as dimensões analisadas.

A etapa seguinte foi a análise da validade convergente, por meio da variância extraída média (AVE). Para o atingimento de valores satisfatórios, procedeu-se exclusão de assertivas das dimensões compromisso com a inovação e comunicação. Desta forma, obteve-se AVE acima de 0,5 para todas as dimensões do estudo.

$\mathrm{Na}$ análise da validade discriminante foram identificados problemas relacionados às dimensões atuação em equipe e compromisso com a inovação. Para a solução de tais problemas foi eliminada a assertiva 4 da dimensão atuação em equipe e do construto compromisso com a inovação. Com os ajustes mencionados, os valores da raiz quadrada da AVE para cada uma das dimensões mantiveram-se maiores do que os coeficientes de correlação entre as variáveis latentes utilizadas, obtendo-se validade discriminante.

A Tabela 5 evidencia os valores de confiabilidade obtidos. 
Tabela 5 - Valores de confiabilidade

\begin{tabular}{c|c|c|c|c}
\hline Dimensão da Inovação & Sigla & AC & CC & AVE \\
\hline Comunicação & COMUN & 0,6 & 0,8 & 0,6 \\
Ambiente físico & AMBFIS & 0,8 & 0,9 & 0,6 \\
Inovação e tecnologia & INOTEC & 0,8 & 0,9 & 0,7 \\
Pessoas & PESS & 0,8 & 0,8 & 0,5 \\
Aprendizagem organizacional & APORG & 0,6 & 0,8 & 0,5 \\
Meio ambiente & MAMBI & 0,9 & 0,9 & 0,7 \\
Atuação em equipe & ATEQUI & 0,9 & 0,9 & 0,8 \\
Estrutura organizacional & ESTORG & 0,6 & 0,8 & 0,6 \\
\hline
\end{tabular}

Fonte: elaboração própria.

Finalizada a análise do modelo de mensuração e atingidos os pressupostos necessários para a utilização da modelagem de equações estruturais, parte-se para a análise do modelo estrutural.

Primeiramente, apresenta-se os valores de $\mathrm{R}^{2}$, que indicam a explicação, pelo modelo estrutural, da variância das variáveis com efeito médio (acima de 0,13) e grande (acima de 0,26), conforme preconiza Cohen (1988). Os dados são apresentados na Tabela 6.

Tabela 6 - Coeficiente de determinação de Pearson $\left(\mathrm{R}^{2}\right)$

\begin{tabular}{c|c|c}
\hline Variáveis dependentes & Sigla & $\mathbf{R}^{\mathbf{2}}$ \\
\hline Custeio baseado em atividades & ABC & 0,250 \\
Gestão baseada em atividades & ABM & 0,314 \\
Custeio por absorção & ABSOR & 0,222 \\
Margem de contribuição & CONTRIB & 0,113 \\
Kaizen & KAIZEN & 0,304 \\
Custeio meta & META & 0,316 \\
Custo padrão & PADRAO & 0,236 \\
Custos da qualidade & QUALID & 0,415 \\
Custeio variável & VARIAVEL & 0,118 \\
Incubadora & INCUB & 0,130 \\
\hline
\end{tabular}

Fonte: elaboração própria.

Dessa forma, pode-se dizer que as práticas de gestão baseada em atividades, kaizen, custeio meta e custos da qualidade possuem maior tendência a serem utilizadas em decorrência da inovação do que as demais práticas. Esses achados podem estar relacionados com os aspectos inerentes a inovação como, por exemplo, a melhoria contínua proposta pelo kaizen, que se alinha à busca por aprimoramento e desenvolvimento inerente ao aspecto inovativo.

Após a aplicação do bootstrapping, identificou-se as significâncias das relações entre os construtos analisados, conforme evidenciado na Tabela 7. Adicionalmente, são evidenciados os coeficientes do modelo ajustado. 
Tabela 7 - Significância e coeficientes das relações do modelo

\begin{tabular}{|c|c|c|c|c|c|c|c|c|c|}
\hline \multicolumn{3}{|c|}{ Variáveis } & \multirow{2}{*}{$\begin{array}{c}\text { Coefic } \\
-0,048\end{array}$} & \multirow{2}{*}{$\frac{\text { p-valor }}{0,695}$} & \multicolumn{3}{|c|}{ Variáveis } & \multirow{2}{*}{$\begin{array}{c}\text { Coefic } \\
-0,203\end{array}$} & \multirow{2}{*}{$\frac{\text { p-valor }}{0,146}$} \\
\hline AMBFIS & $\rightarrow$ & $A B C$ & & & APORG & $\rightarrow$ & $A B C$ & & \\
\hline AMBFIS & $\rightarrow$ & ABM & $-0,120$ & 0,313 & APORG & $\rightarrow$ & ABM & $-0,112$ & 0,348 \\
\hline AMBFIS & $\rightarrow$ & ABSOR & 0,051 & 0,642 & APORG & $\rightarrow$ & ABSOR & $-0,171$ & 0,285 \\
\hline AMBFIS & $\rightarrow$ & $\begin{array}{l}\text { CON- } \\
\text { TRIB }\end{array}$ & $-0,076$ & 0,507 & APORG & $\rightarrow$ & $\begin{array}{l}\text { CON- } \\
\text { TRIB }\end{array}$ & $-0,152$ & 0,322 \\
\hline AMBFIS & $\rightarrow$ & KAIZEN & $-0,076$ & 0,455 & APORG & $\rightarrow$ & KAIZEN & $-0,140$ & 0,272 \\
\hline AMBFIS & $\rightarrow$ & META & 0,032 & 0,746 & APORG & $\rightarrow$ & META & $-0,016$ & 0,926 \\
\hline AMBFIS & $\rightarrow$ & PADRAO & 0,026 & 0,820 & APORG & $\rightarrow$ & PADRAO & 0,116 & 0,365 \\
\hline AMBFIS & $\rightarrow$ & QUALID & $-0,052$ & 0,599 & APORG & $\rightarrow$ & QUALID & $-0,024$ & 0,830 \\
\hline AMBFIS & $\rightarrow$ & $\begin{array}{l}\text { VARI- } \\
\text { AVEL }\end{array}$ & 0,118 & 0,375 & APORG & $\rightarrow$ & $\begin{array}{l}\text { VARI- } \\
\text { AVEL }\end{array}$ & 0,170 & 0,373 \\
\hline AMBFIS & $\rightarrow$ & INCUB & $-0,019$ & 0,866 & APORG & $\rightarrow$ & INCUB & 0,018 & 0,905 \\
\hline ATEQUI & $\rightarrow$ & $A B C$ & $-0,019$ & 0,910 & COMUN. & $\rightarrow$ & $A B C$ & $-0,075$ & 0,616 \\
\hline ATEQUI & $\rightarrow$ & ABM & $-0,218$ & 0,183 & COMUN. & $\rightarrow$ & ABM & 0,074 & 0,612 \\
\hline ATEQUI & $\rightarrow$ & ABSOR & 0,005 & 0,977 & COMUN. & $\rightarrow$ & ABOSR & $-0,148$ & 0,321 \\
\hline ATEQUI & $\rightarrow$ & $\begin{array}{l}\text { CON- } \\
\text { TRIB }\end{array}$ & $-0,137$ & 0,410 & COMUN. & $\rightarrow$ & $\begin{array}{l}\text { CON- } \\
\text { TRIB }\end{array}$ & $-0,052$ & 0,748 \\
\hline ATEQUI & $\rightarrow$ & KAIZEN & $-0,109$ & 0,457 & COMUN. & $\rightarrow$ & KAIZEN & 0,027 & 0,861 \\
\hline ATEQUI & $\rightarrow$ & META & 0,036 & 0,815 & COMUN. & $\rightarrow$ & META & 0,293 & 0,032 \\
\hline ATEQUI & $\rightarrow$ & PADRAO & 0,163 & 0,328 & COMUN. & $\rightarrow$ & PADRÃO & 0,137 & 0,378 \\
\hline ATEQUI & $\rightarrow$ & QUALID & $-0,053$ & 0,702 & COMUN. & $\rightarrow$ & QUALID & $-0,028$ & 0,840 \\
\hline ATEQUI & $\rightarrow$ & $\begin{array}{l}\text { VARI- } \\
\text { AVEL }\end{array}$ & 0,071 & 0,679 & COMUN. & $\rightarrow$ & $\begin{array}{l}\text { VARI- } \\
\text { AVEL }\end{array}$ & $-0,075$ & 0,663 \\
\hline ATEQUI & $\rightarrow$ & INCUB & 0,135 & 0,446 & COMUN. & $\rightarrow$ & INCUB & 0,397 & 0,006 \\
\hline ESTORG & $\rightarrow$ & $A B C$ & 0,348 & 0,025 & INOTEC & $\rightarrow$ & $A B C$ & $-0,082$ & 0,485 \\
\hline ESTORG & $\rightarrow$ & ABM & 0,267 & 0,045 & INOTEC & $\rightarrow$ & ABM & $-0,072$ & 0,566 \\
\hline ESTORG & $\rightarrow$ & ABOSR & 0,189 & 0,269 & INOTEC & $\rightarrow$ & ABOSR & 0,164 & 0,280 \\
\hline ESTORG & $\rightarrow$ & $\begin{array}{l}\text { CON- } \\
\text { TRIB }\end{array}$ & 0,093 & 0,573 & INOTEC & $\rightarrow$ & $\begin{array}{l}\text { CON- } \\
\text { TRIB }\end{array}$ & 0,111 & 0,475 \\
\hline ESTORG & $\rightarrow$ & KAIZEN & 0,283 & 0,107 & INOTEC & $\rightarrow$ & KAIZEN & $-0,001$ & 0,994 \\
\hline ESTORG & $\rightarrow$ & META & 0,142 & 0,316 & INOTEC & $\rightarrow$ & META & $-0,159$ & 0,254 \\
\hline ESTORG & $\rightarrow$ & PADRÃO & 0,026 & 0,887 & INOTEC & $\rightarrow$ & PADRÃO & $-0,258$ & 0,123 \\
\hline ESTORG & $\rightarrow$ & QUALID & 0,027 & 0,835 & INOTEC & $\rightarrow$ & QUALID & 0,000 & 0,997 \\
\hline ESTORG & $\rightarrow$ & $\begin{array}{l}\text { VARI- } \\
\text { AVEL }\end{array}$ & 0,134 & 0,405 & INOTEC & $\rightarrow$ & $\begin{array}{l}\text { VARI- } \\
\text { AVEL }\end{array}$ & 0,051 & 0,727 \\
\hline ESTORG & $\rightarrow$ & INCUB & $-0,085$ & 0,526 & INOTEC & $\rightarrow$ & INCUB & $-0,149$ & 0,277 \\
\hline MAMBI & $\rightarrow$ & $A B C$ & 0,325 & 0,012 & PESS & $\rightarrow$ & $A B C$ & 0,167 & 0,290 \\
\hline
\end{tabular}




\begin{tabular}{|c|c|c|c|c|c|c|c|c|c|}
\hline \multicolumn{3}{|c|}{ Variáveis } & \multirow{2}{*}{$\begin{array}{c}\text { Coefic } \\
0,225\end{array}$} & \multirow{2}{*}{$\frac{\text { p-valor }}{0,102}$} & \multicolumn{3}{|c|}{ Variáveis } & \multirow{2}{*}{$\begin{array}{c}\text { Coefic } \\
0,447\end{array}$} & \multirow{2}{*}{$\frac{\text { p-valor }}{0,005}$} \\
\hline MAMBI & $\rightarrow$ & ABM & & & PESS & $\rightarrow$ & ABM & & \\
\hline MAMBI & $\rightarrow$ & ABOSR & 0,373 & 0,006 & PESS & $\rightarrow$ & ABOSR & $-0,012$ & 0,939 \\
\hline MAMBI & $\rightarrow$ & $\begin{array}{l}\text { CON- } \\
\text { TRIB }\end{array}$ & $-0,023$ & 0,88 & PESS & $\rightarrow$ & $\begin{array}{l}\text { CON- } \\
\text { TRIB }\end{array}$ & 0,418 & 0,020 \\
\hline MAMBI & $\rightarrow$ & KAIZEN & 0,332 & 0,013 & PESS & $\rightarrow$ & KAIZEN & 0,233 & 0,148 \\
\hline MAMBI & $\rightarrow$ & META & 0,113 & 0,405 & PESS & $\rightarrow$ & META & 0,218 & 0,175 \\
\hline MAMBI & $\rightarrow$ & PADRÃO & 0,261 & 0,047 & PESS & $\rightarrow$ & PADRÃO & 0,068 & 0,648 \\
\hline MAMBI & $\rightarrow$ & QUALID & 0,500 & 0,000 & PESS & $\rightarrow$ & QUALID & 0,301 & 0,018 \\
\hline MAMBI & $\rightarrow$ & $\begin{array}{l}\text { VARI- } \\
\text { AVEL }\end{array}$ & 0,056 & 0,711 & PESS & $\rightarrow$ & $\begin{array}{l}\text { VARI- } \\
\text { AVEL }\end{array}$ & $-0,150$ & 0,404 \\
\hline MAMBI & $\rightarrow$ & INCUB & $-0,219$ & 0,076 & PESS & $\rightarrow$ & INCUB & 0,049 & 0,756 \\
\hline INCUB & $\rightarrow$ & $\mathrm{ABC}$ & 0,074 & 0,434 & INCUB & $\rightarrow$ & META & $-0,168$ & 0,080 \\
\hline INCUB & $\rightarrow$ & $\mathrm{ABM}$ & 0,019 & 0,838 & INCUB & $\rightarrow$ & PADRAO & $-0,043$ & 0,674 \\
\hline INCUB & $\rightarrow$ & ABSOR & 0,112 & 0,262 & INCUB & $\rightarrow$ & QUALID & $-0,149$ & 0,072 \\
\hline INCUB & $\rightarrow$ & $\begin{array}{l}\text { CON- } \\
\text { TRIB }\end{array}$ & 0,042 & 0,700 & INCUB & $\rightarrow$ & $\begin{array}{l}\text { VARI- } \\
\text { AVEL }\end{array}$ & 0,112 & 0,313 \\
\hline INCUB & $\rightarrow$ & KAIZEN & 0,003 & 0,973 & & & & & \\
\hline
\end{tabular}

Fonte: elaboração própria.

Em termos de significância, considera-se, nesta pesquisa, 10\%, alcançando um nível de confiança de $90 \%$. Observa-se que as dimensões da organização que possuem relações mais significantes diretamente com as práticas de gestão de custos são meio ambiente, pessoas, estrutura organizacional e comunicação. Além disso, a incubação das empresas apresenta relação significativa apenas com custeio meta e custos da qualidade.

Quanto às dimensões com significância para a adoção de práticas de gestão de custos, estas estão diretamente ligadas às pessoas da organização, também com o processo de comunicação, flexibilidade na execução das tarefas, tomada de decisões e, ainda, preocupação com aspectos ambientais. Portanto, quanto mais as organizações atendem as dimensões de (i) meio ambiente, com foco na preocupação com aspectos ambientais, conscientização e redução de consumo, (ii) pessoas, motivando seus colaboradores, treinando-os e incentivando-os para a criatividade, (iii) estrutura organizacional, a partir de um ambiente descentralizado e com foco na melhoria de processos, e (iv) comunicação, com veículos de comunicação interna e ampla divulgação das informações, maior tende a ser a adoção de práticas de gestão de custos.

Em termos da passagem das empresas por processo de incubação, este não apresenta, portanto, relação significativa com a maioria das práticas de gestão de custos. Este resultado alinha-se ao encontrado por Raupp (2011), onde a minoria das empresas incubadas analisadas possuía formalização do controle de custos.

Ainda na pesquisa de Raupp (2011), o autor identificou que as incubadoras disponibilizam suporte à gestão de custos das empresas analisadas. No entanto, infere-se, a partir desta pesquisa, que este suporte não se refere às práticas de gestão de custos, não abarcando, portanto, aspectos relacionados a gestão de custos para suporte à tomada de decisões por parte das empresas ou, ainda, que o treinamento ofertado não tem sido efetivo a ponto de que as empresas percebam a utilidade das práticas e as adotem. Salienta-se, no entanto, que as práticas com significância para a passagem pelo processo de incubação são custeio meta e custos da qualidade.

Em relação aos coeficientes do modelo, observa-se que a maior parte das práticas de gestão de custos são influenciadas de forma positiva pelas dimensões da organização inovadora, sendo que as dimensões que influenciam a maior parte das práticas são estrutura organizacional, meio ambiente, pessoas e comunicação. Estas dimensões, além de influenciarem positivamente a adoção das práticas de gestão de custos, o fazem de forma significativa. Quanto à passagem das empresas por processo de incubação, esta possui relação positiva com a adoção da maior parte das práticas de gestão de custos analisadas, com exceção de custeio meta, custo padrão e custos da qualidade, que apresentaram relação inversa.

Para facilitar a visualização dos resultados, a Figura 1 apresenta, de forma simplificada, em decorrência do número de variáveis, os coeficientes das relações significativas identificadas. 
Figura 1 - Coeficientes das relações com significância

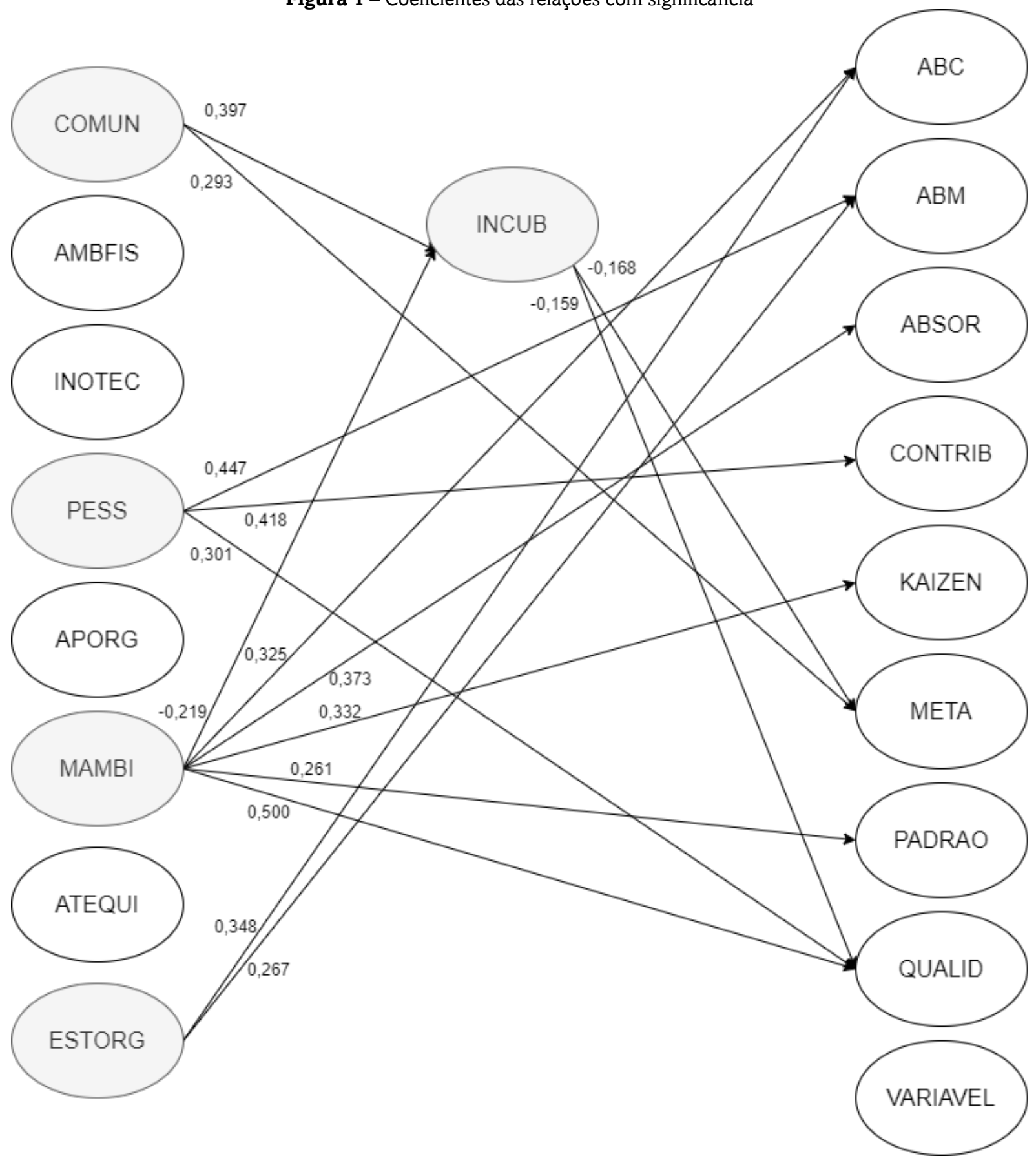

Fonte: elaboração própria.

Quanto ao efeito mediador exercido pela passagem por incubadora de empresas, considerando a baixa significância da incubação para as práticas de gestão de custos, bem como das dimensões da organização inovadora para a incubação, acrescido ainda do baixo poder explicativo e, em alguns casos, inverso da incubação para as práticas, não se pode aceitar a hipótese de que a passagem das empresas por um processo de incubação poderia mediar a adoção de práticas de gestão de custos em organizações inovadoras, influenciando, de forma conjunta, a adoção de tais práticas. Este resultado se alinha ao já exposto, onde apesar de haver o entendimento de que um dos suportes ofertado pelas incubadoras de empresas é o gerencial, este pode não estar sendo efetivo. 


\section{CONSIDERAÇÕES FINAIS}

Este estudo discute a adoção de práticas de gestão de custos, passagem por incubadoras e inovação nas organizações, de forma a analisar a influência das dimensões da organização inovadora na adoção de práticas de gestão de custos mediada pelo processo de incubação de empresas. A partir disso, a hipótese estabelecida foi a de que a passagem das empresas por um processo de incubação pode mediar a adoção de práticas de gestão de custos em organizações inovadoras, influenciando, de forma conjunta, a adoção de tais práticas.

Identificou-se baixa utilização de práticas modernas de gestão de custos nas empresas da amostra, sendo utilizadas com maior intensidade as práticas tradicionais, com destaque para a margem de contribuição. Este resultado indica que não estão sendo introduzidas intensamente, nas empresas estudadas, práticas surgidas mais recentemente na literatura.

Destaca-se que a baixa utilização da prática de custeio baseado em atividades torna-se um contraponto com a literatura recente, que tem publicado pesquisas com a utilização desta prática (Melo \& Leone, 2015; Santana et al., 2015; Silva et al., 2015; Nowak \& Linder, 2016; Rezyapova, 2016; Zambon \& Bee, 2016; Lima \& Azzolini Júnior, 2017; Zhou et al., 2018), enquanto a realidade das empresas analisadas é de baixa intensidade de aplicação do ABC.

Em relação às dimensões da organização inovadora, identificou-se que algumas possuem relações mais significativas com a adoção das práticas de gestão de custos do que outras. Este fato decorre das características destas dimensões, dado que as relacionadas à comunicação dentro da organização, à maior flexibilidade em termos decisórios, ao meio ambiente e às pessoas têm capacidade de influenciar as práticas de gestão de custos com maior significância e este fato por estar relacionado com a maior flexibilidade e abertura que tais dimensões representam para os colaboradores na sua relação com a organização, que permitiria maior interação e aceitação a mudanças nas práticas adotadas. Quanto ao processo de incubação, este não se apresentou significante para a adoção da maioria das práticas de gestão de custos, indicando que a adoção de tais práticas, pelas empresas analisadas, não decorre de sua passagem por um processo de incubação e que este processo, portanto, não tem dado suporte para o desenvolvimento, dentro das empresas, de aparato gerencial em termos de práticas de gestão de custos.

No que diz respeito ao efeito mediador exercido pela passagem por processo de incubação, considerando a baixa significância deste processo na adoção das práticas de gestão de custos, acrescido ao baixo poder explicativo e, em determinados casos, inverso, que este processo exerce sobre a adoção de tais práticas, não se pode aceitar a hipótese de que a passagem das empresas por um processo de incubação poderia mediar a adoção de práticas de gestão de custos em organizações inovadoras.

Neste sentido, infere-se que, na amostra analisada, as empresas podem não ter recebido suporte gerencial das incubadoras de empresas no que diz respeito à gestão de custos e à adoção e implementação de práticas que deem suporte à tomada de decisão.

Este estudo fornece insights para novas investigações sobre a baixa adoção de práticas modernas de gestão de custos, que pode estar relacionada não somente às características das práticas em si, como as dificuldades relacionadas à implementação do ABC, por exemplo, mas, sobretudo, com a característica das organizações que as adotam, se inovadoras ou não, por exemplo, e também com o ambiente onde estão inseridas, tal como o de incubação, indicando a necessidade de análises conjuntas da adoção com outros fatores influenciadores desta relação.

Ao avaliar os resultados desta pesquisa, devem ser consideradas algumas limitações, que podem indicar lacunas para futuros estudos na área. Entre estas limitações, pode-se citar o espaço temporal utilizado como base para a identificação das pesquisas de gestão de custos e das práticas mencionadas, a utilização de empresas que receberam auxílio financeiro para desenvolvimento de inovações, bem como o uso de resposta dicotômica acerca do processo de incubação. Assim, sugere-se que, em futuras investigações, seja ampliado o espaço temporal das pesquisas de gestão de custos para a identificação das práticas em um período maior de tempo, além da utilização de amostra diferente, como o uso de empresas que sejam reconhecidamente inovadoras, de forma a identificar como esta característica pode influenciar seus processos de gestão. Sugere-se, ainda, que seja analisada em que medida as incubadoras têm fornecido apoio para a adoção de práticas de gestão de custos.

\section{REFERÊNCIAS}

Abdel-Kader, M., \& Luther, R. (2006). IFAC's conception of the evolution of management accounting: A research note. Advances in Management Accounting, 229-247. https://doi.org/10.1016/S1474-7871(06)15010-8

Abib, G., Hayashi Junior, P., Gomel, M. M., \& Fonseca, M. W. (2013). O papel construtivo das incubadoras no alinhamento estratégico e mercadológico das empresas incubadas e graduadas. Revista de Empreendedorismo e Gestão de Pequenas Empresas, 1(2), 33-59.

Aguilà, S. (2016). En busca de la suficiencia financiera en la universidad pública española: De la financiación al control y la gestión de costes. Intangible Capital, 12(1), 17-50.

Arabi, M., \& Kavianifard, H. (2013). Examining the relation between stage of management accounting practice and technological innovation and management accounting innovation in the companies listed in Tehran Stock Exchange. Advances in Environmental Biology, $7(10 \mathrm{~S} 1), 3145-3160$.

Associação Nacional de Entidades Promotoras de Empreendimentos Inovadores - ANPROTEC (2012). Estudo, Análise e Proposições Sobre as Incubadoras de Empresas no Brasil. Brasília. 
Baharudin, N., \& Jusoh, R. (2015). Target Cost Management (TCM): a case study of an automotive company. Procedia-Social and Behavioral Sciences, 172, 525-532.

Baird, K. (2007). Adoption of activity management practices in public sector organizations. Accounting \& Finance, 47(4), 551-569. https://doi.org/10.1111/j.1467-629X.2007.00225.x

Baird, K. M., Harrison, G. L., \& Reeve, R. C. (2004). Adoption of activity management practices: a note on the extent of adoption and the influence of organizational and cultural factors. Management Accounting Research, 15(4), 383-399. https://doi.org/10.1016/j. mar.2004.07.002

Bautzer, D. (2009). Inovação: repensando as organizações. São Paulo: Atlas.

Ben, F., \& Canossa, F. (2017). Cost and profitability analysis between Pinot Noir and BRS Carmen grapes production at a family property in Monte Belo do Sul-RS. Custos e Agronegócio On Line, 13(3), 340-376.

Bokor, Z., \& Markovits-Somogyi, R. (2015). Improved cost management at small and medium sized road transport companies: case Hungary. Promet-Traffic\&Transportation, 27(5), 417-428.

Bonacim, C. A. G., \& de Araujo, A. M. P. (2010). Gestão de custos aplicada a hospitais universitários públicos: a experiência do Hospital das Clínicas da Faculdade de Medicina de Ribeirão Preto da USP. Revista de Administração Pública, 44(4), 903-931.

Braga, D. P. G., Braga, A. X. V., \& Souza, M. A. (2010). Gestão de Custos, Preços e Resultados: um Estudo em Indústrias Conserveiras do Rio Grande do Sul. Revista Contabilidade, Gestão e Governança, 13(2).

Braga, L. N. P., \& Raupp, F. M. (2020). Gestão de Custos Alinhada à Tomada de Decisão: o Caso de uma Empresa Incubada de Base Tecnológica. Revista de Negócios, 25(3), 22-44.

Broze, T. S., Facó, R. T., dos Santos Silva, L. C., de Souza, J. S., \& Ten Caten, C. S. (2019). Fatores que influenciam o desenvolvimento de empresas incubadas: uma pesquisa de perspectiva/Factors that influence the development of incubated enterprises: a perspective research. Brazilian Journal of Business, 1(2), 500-514.

Charaf, K., \& Bescos, P. L. (2013). The role of organizational and cultural factors in the adoption of activity-based costing: the case of Moroccan firms. Accounting and Management Information Systems, 12(1), 4.

Chenhall, R. H., \& Langfield-Smith, K. (1998). Adoption and benefits of management accounting practices: an Australian study. Management Accounting Research, 9(1), 1-19. https://doi.org/10.1006/mare.1997.0060

Chenhall, R. H., \& Moers, F. (2015). The role of innovation in the evolution of management accounting and its integration into management control. Accounting, Organizations And Society, 47, 1-13. https://doi.org/10.1016/j.aos.2015.10.002

Cohen, J. (2013). Statistical power analysis for the behavioral sciences. Routledge.

Cooper, D. R., \& Schindler, P. S. (2004). Métodos de pesquisa em administração. Porto Alegre: Bookman.

Costa, A. H. da. (2011). Análise comparativa das principais características das pesquisas de inovação tecnológica de Brasil e Espanha. Dissertação (Mestrado em Engenharia de Produção) - Universidade Federal da Paraíba, João Pessoa, Brasil.

Engelage, E., Borgert, A., Gasparetto, V., Lunkes, R. J., \& Schnorrenberger, D. (2017). Cost management in green logistics: analysis in an agro-industry. Custos e Agronegócio On Line, 13(1), 174-376.

Fernandes, C. C. (2014). Análise estratégica da contribuição das incubadoras de empresas de base tecnológica para as empresas incubadas. Dissertação (Mestrado em Administração) - Faculdade de Economia, Administração e Contabilidade da Universidade de São Paulo, São Paulo, Brasil, 2014.

Ferreira, M. P., de Abreu, A. F., de Abreu, P. F., Trzeciak, D. S., Apolinário, L. G., \& da Cunha, A. D. A. (2008). Gestão por indicadores de desempenho: resultados na incubadora empresarial tecnológica. Production, 18(2), 302-318.

Galbraith, J. R., \& Kazanjian, R. K. (1986). Strategy implementation: structure, systems and processes. In: Grant, R. M. (2010). Contemporary strategy analysis. Malden: Blackwell.

Govindarajan, V:; Trimble, C. (2006). Os dez mandamentos da inovação estratégica. Rio de Janeiro: CAMPUS.

Hair, J. F., Black, W. C., Babin, B. J., Anderson, R. E., \& Tatham, R. L. (2009). Análise multivariada de dados. Bookman Editora.

Iacono, A., Almeida, C. A. S. D., \& Nagano, M. S. (2011). Interação e cooperação de empresas incubadas de base tecnológica: uma análise diante do novo paradigma de inovação. Revista de Administração Pública, 45(5), 1485-1516.

Imlau, J.M. \& Gasparetto, V. (2017). Práticas de contabilidade gerencial em cooperativas de produção agropecuária do Estado do Rio Grande do Sul. Custos e Agronegócio On Line, 13 (2), 23-264.

International Federation Of Accountants (IFAC) (1998). International Management Accounting Practice Statement: Management Accounting Concepts. New York.

Joshi, P. L. (2001). The international diffusion of new management accounting practices: the case of India. Journal of International Accounting, Auditing and Taxation, 10(1), 85-109. https://doi.org/10.1016/S1061-9518(01)00037-4

Jurčević, M., Bubalo, T., \& Mandžuka, B. (2018). Influence of costs on the optimization of transport routes (case study)-Passenger transportation company from Zagreb. Ekonomski Vjesnik, 31(1), 65-73.

Lazzarotti, F., da Silveira, A. L. T., Carvalho, C. E., Rossetto, C. R., \& Sychoski, J. C. (2015). Orientação empreendedora: um estudo das dimensões e sua relação com desempenho em empresas graduadas. RAC-Revista de Administração Contemporânea, 19(6), 673-695.

Lima, L. M., \& Júnior, W. A. (2017). Activity Based Costing (ABC) in the commercial coffee sector of a large company. Custos e Agronegócio On Line, 13(1), 206-239. 
Machado, D. G., Reckziegel, V., Souza, M. A. de, \& Almeida, L. B. de (2016). Gestão de Custos da Logística Verde: Análise Exploratória das Contribuições Empírico-Teóricas de Pesquisa. Contabilidade Vista \& Revista, 27(2), 88-112.

Mantri, S., \& Jaju, S. (2017). Cost of quality management in indian industries: a practical insight. International Journal for Quality Research, 11(3).

Melo, M. A. de, \& Leone, R. J. G. (2015). Alinhamento entre as estratégias competitivas e a gestão de custos: um estudo em pequenas empresas industriais do setor de transformação. BBR-Brazilian Business Review, 12(5), 83-104. http://dx.doi.org/10.15728/ bbr.2015.12.5.5

Morais, O. de O., Coelho, A. C. D., \& Holanda, A. P. (2014). Artefatos de contabilidade gerencial e maximização do valor em firmas brasileiras. Revista Contabilidade e Controladoria, 6(2), 128-146.

Naranjo-Gil, D., Maas, V. S., \& Hartmann, F. G. (2009). How CFOs determine management accounting innovation: an examination of direct and indirect effects. European Accounting Review, 18(4), 667-695. https://doi.org/10.1080/09638180802627795

Nowak, C., \& Linder, C. (2016). Do you know how much your expatriate costs? An activity-based cost analysis of expatriation. Journal of Global Mobility, 4(1), 88-107. https://doi.org/10.1108/JGM-10-2015-0043

Oliveira, J. N. (2006). Características e práticas gerenciais de empresas inovadoras: um estudo de caso numa empresa do setor têxtil do Estado da Paraiba. Dissertação (Mestrado em Engenharia de Produção) - Universidade Federal da Paraíba, João Pessoa, Brasil.

Raupp, F. M. (2011). Gestão de custos nas empresas incubadas em incubadoras brasileiras. Revista Gestão Industrial, 7(2).

Raupp, F. M., \& Beuren, I. M. (2006). O suporte das incubadoras brasileiras para potencializar as características empreendedoras nas empresas incubadas. Revista de Administração-RAUSP, 41(4), 419-430.

Rezyapova, A. (2016). Efficiency improvement of menegment's process cost menegment in network retail businesses. International Journal for Quality Research, 10(4). https://doi.org/10.18421/IJQR10.04-06

Rocha, D. T. da, Souza, A., Cruz, J. A. W., \& Tracz, L. (2010). Gestão de custos: um estudo bibliométrico e sociométrico da produção científica do EnANPAD 1997-2008. In Anais do Congresso Brasileiro de Custos-ABC. Belo Horizonte, MG.

Rothwell, R. Policies in industry. In Pavitt, K. (1980). Technical innovation and British economic performance. London: The Macmillan Press.

Santa Rita, C. (2017). O papel das incubadoras de empresas de base tecnológica no desenvolvimento regional: "moda" ou "realidade"? Dissertação (Mestrado Profissional em Administração) - Faculdades Integradas de Pedro Leopoldo, Belo Horizonte, Brasil.

Santana, A. F. B., Medeiros, H. da S., \& Guimarães, L. da S. (2015). Uma nova abordagem do Activity-Based Costing-ABC: desenvolvimento e aplicação do abc matricial em uma indústria calçadista de Portugal. Exacta, 13(3), 315-325. https://doi.org/10.5585/ ExactaEP.v13n3.5773

Santos, V. D., Dorow, D. R., \& Beuren, I. M. (2016). Práticas gerenciais de micro e pequenas empresas. Revista Ambiente Contábil, 8(1), 153-186.

Santos, V. dos Beuren, I. M., \& Conte, A. (2017). Uso de instrumentos do sistema de controle gerencial em empresas incubadas. Contabilidade Vista \& Revista, 28(3), 103-132.

Silva, G. R. da, Santos Neto, E. M. dos, Araújo, M. A. de, \& Ceolin, A. C. (2016). Corporate Controllership and price formation from the light of cost management: a case study of a Paraiba State supermarket. Custos e Agronegocio On Line, 12(2), 352-373.

Silva, P., Gasparetto, V., \& Lunkes, R. J. (2015). Custos no Transporte Rodoviário de Passageiros e Encomendas: estudo em uma empresa catarinense. Revista Catarinense da Ciência Contábil, 14(42), 25-40. http://dx.doi.org/10.16930/2237-7662/rccc.v14n42p25-40

Soutes, D. O. (2006). Uma investigação do uso de artefatos da contabilidade gerencial por empresas brasileiras (Doctoral dissertation). Available from http://www.teses.usp.br/teses/disponiveis/12/12136/tde-12122006-102212/pt-br.php

Souza, F. F. (2017). Relação entre inovação e adoção de práticas modernas de contabilidade gerencial. Dissertação (Mestrado em Contabilidade) - Universidade Federal de Santa Catarina, Florianópolis, Brasil.

Tidd, J., Bessant, J., \& Pavitt, K. Gestão da inovação. 3. ed. Porto Alegre: Bookman.

Tiscoski, G. P. (2015). O processo de aprendizagem organizacional em uma incubadora social. Tese (Doutorado em Administração) Faculdade de Economia, Administração e Contabilidade da Universidade de São Paulo, São Paulo, Brasil.

Uyar, A. (2010). Cost and management accounting practices: a survey of manufacturing companies. Eurasian Journal of Business and Economics, 3(6), 113-125

Vargas, S. B. de, Coser, T., \& Souza, M. A. de (2016). Mensuração dos Custos Logísticos: Estudo de Caso em uma Indústria Gráfica. Contabilidade Vista \& Revista, 27(1), 63-87.

Volpato, M., \& Cimbalista, S. (2002). O processo de motivação como incentivo à inovação nas organizações. Revista da FAE, 5(3), 75-86.

Wrubel, F., Diehl, C. A., Toigo, L. A., \& Ott, E. (2011). Uma proposta para a validação de categorias sobre gestão estratégica de custos. Revista Brasileira de Gestão de Negócios-RBGN, 13(40), 332-348.

Zambon, E. P., \& Bee, D. (2016). Cost management in agribusiness: the use of Activity Based Cost (ABC) in a small rural property. Custos e Agronegocio On Line, 12(3), 137-152.

Zhou, W., Li, Y., Liu, H., \& Song, X. (2018). The Cost management on the quantification of responsibility. Mathematical Problems in Engineering, 2018. https://doi.org/10.1155/2018/1762798

Zimon, G. (2016). Accounting tools vs. logistics costs control in a trading company. LogForum, 12. 